\title{
Erratum
}

\section{MASS-GENERATION, SYMMETRY BREAKING AND CONFORMAL INVARIANCE}

M. Flato, M. Guenin: Czech. J. Phys. B32 (1982) 490

Formula on p. 490, line $18 \Omega(a, x)=1 /\left(1+2 a \cdot x+a^{0} x^{0}\right)$

should read

$\Omega(a, x)=1 /\left(1+2 a \cdot \mathbf{x}+a^{2} x^{2}\right)$

Page 490, line 21

It is true, in general, of all massless ...

replace by

It is true, in general, of most massless ...

Page 490, lines 27, 28 Notice that even in the case $m$ is a constant ...

replace by

Notice that even in the case when $m$ is a constant ...

Page 490, lines 33, 34 This procedure recalls that ...

replace by

This procedure reminds that ...

Page 491, line 27

coupling constants with dimensions in $\Phi^{4}$ theory, in the Fermi-coupling, etc.

replace by

coupling constants with dimensions in $\Phi^{n}$ theory $(n \neq 4)$ in the 4-Fermi-coupling, etc.

Page 491, the expression in the last but one equation

$$
-\mathrm{i} \varepsilon \phi^{*} \partial^{\mu} \phi
$$

replace by

$$
-\frac{1}{2} i \varepsilon \phi^{*} \partial^{\mu} \phi
$$

Page 491, the expression in the last equation

replace by

$$
\lambda \bar{\psi} \frac{\phi}{\sqrt{ }(\phi \phi)} \psi-\varepsilon^{2} A_{\mu} A^{\mu} \phi+i \varepsilon A^{\mu} \partial_{\mu} \phi
$$

$$
\lambda \bar{\psi} \frac{\phi}{\sqrt{ }\left(\phi^{*} \phi\right)} \psi-\varepsilon^{2} A_{\mu} A^{\mu} \phi
$$

